\title{
Searching for Fast Optical Transients using a VERITAS Cherenkov Telescope
}

\author{
Sean C. Griffin \\ Physics Department, McGill University, \\ 3600 University Street, Montreal, QC, H3A2T8, Canada \\ email: griffins@physics.mcgill.ca
}

\begin{abstract}
Astronomical transients are intrinsically interesting objects to study. However, fast optical transients ( $\mu$ s time-scales) are a largely unexplored field of optical astronomy. Most optical observations use instruments that have integration times of the order of seconds and are thus unable to resolve fast transients. Current-generation atmospheric Cherenkov gammaray telescopes such as VERITAS, which consists of four 12-m telescopes, have huge collecting areas, much larger than those of any existing optical telescopes. This paper outlines the benefits of using a Cherenkov telescope to detect optical transients, and the implementation of the VERITAS Transient Detector (TRenDy), a dedicated multi-channel photometer based on fieldprogrammable gate arrays which can be used on VERITAS for such studies without interfering with gamma-ray observations.
\end{abstract}

Keywords. accretion, instrumentation: photometers, X-rays: binaries

\section{Introduction}

In optical astronomy, fine time-resolution observations on a $\mu$ s time-scale are rare, owing to the fact that most optical detectors (such as CCDs) have long integration times, on the scale of seconds or longer. Moreover, because large optical telescopes are oversubscribed, few attempts have been made to search for fast optical transients.

Atmospheric Cherenkov detectors such as VERITAs (Holder et al. 2006) have huge collecting areas (at the cost of angular resolution), and the nature of gamma-ray observations make observing under moonlight difficult or impossible. Thus, time is usually available for other studies. H.E.S.S. has carried out a search for fast optical transients using a purposebuilt camera built specifically for this search (Deil et al. 2009). This paper describes the VERITAS Transient Detector (TRenDy): a high time-resolution, multi-channel, photon counting rate-meter. TRenDy was designed to be integrated into the standard VERITAS telescope readout system, thus making the transition from gamma-ray to optical observing rapid. The limits of the sensitivity of TRenDy due to background light can be traded against gains made by extended observations.

The primary source candidates are X-ray binary systems: the emission mechanism for fast optical transients is probably the accretion of matter onto compact objects. Compact objects such as black holes and neutron stars have sizes of 10s of kilometres, which suggests typical time-scales of 10-100 $\mu$ s. Millisecond time-scale optical variability has already been observed in X-ray binaries (Kanbach et al. 2001; Baratolini et al. 1994) and pulsars (Shearer et al. 2003). The X-ray binary Cygnus X-1 has been observed by Dolan (2001) to emit pulses in the UV with durations of the order of $\leqslant 10 \mathrm{~ms}$, a timescale which requires the production mechanism to be somewhere in the system's accretion disk. That agrees with measurements of X-ray sources GX339-4 (Fabian et al. 1982) and V4641 Sagittarii (Uemura et al. 2002). Fluctuations in the optical afterglow of gamma-ray 

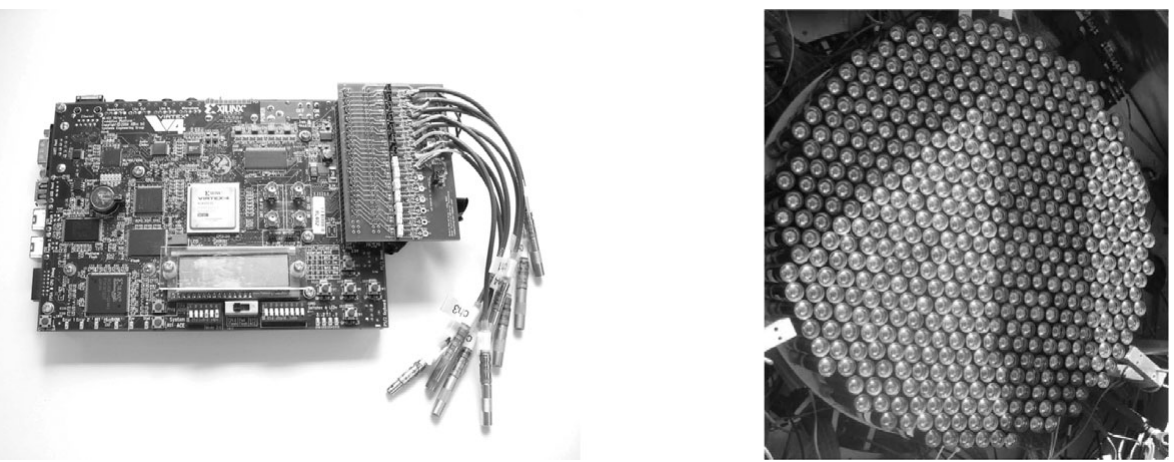

Figure 1. Left: The TRenDy rate meter. The design is based on the Xilinx ML402 FPGA board and contains a Virtex-4 XC4VSX35-FF668-1 FPGA (centred). The custom mezzanine board (right of the FPGA) acts as an interface between the VERITAS CFDs and the FPGA. The LEMO cables to the right connect to the standard VERITAS readout chain. Right: The VERITAS camera is made up of 499 photomultiplier tubes with attached light cones (not shown here) to minimize dead space and block light not coming from the VERITAS reflector. The hexagonal packing leads to a natural subset of the central seven PMTs being used by TRenDy.

bursts, on time-scales of less than an hour, have also been detected (Bersier et al. 2003). It may be possible to see fine structure in the light curves of such transients.

\section{The Veritas Transient Detector}

The TRenDy rate-meter is built from the commercially available Xilinx ML402 Evaluation Platform FPGA board. It also contains a custom-built mezzanine board for loading NIM signals from the constant-fraction discriminators (CFDs) of the standard VERITAS readout into the FPGA. A photo of TRenDy is shown in Fig. 1 (left).

The device has seven channels corresponding to the centre seven pixels of the VERITAS camera (Fig. 1, right) and is designed to use the centre pixel to collect light from the target source and six channels around it to form a "veto ring". The point spread function of a VERITAs telescope is less than the field of view of a single camera pixel $\left(0^{\circ} .15\right)$ : all light from a point source will be well contained within the centre pixel. The six channels of the ring provide a measure of the background rates, but also the ability to veto non-astronomical events: if a signal is seen in both the veto ring and the central pixel simultaneously it must be from a terrestrial source, such as lightning. Similarly, if a signal is seen to move from pixel to pixel, the event can also be vetoed; possible sources of this type are aeroplanes, meteors or spacecraft.

\section{Tests and Results}

One test performed to verify the functionality of TRenDy was the observation of the transit of the image of a bright star across the PMTs of the Whipple 10-m telescope (Kildea et al. 2007). The telescope was slewed to a position ahead of the target star in right ascension. The telescope's tracking system was then disengaged, causing the star's image to drift across the face of the camera. The effect is clearly seen in Fig. 2 (left). A second test was the observation of the Crab Optical Pulsar. The light curve shown in Fig. 2 (right) was reconstructed from TRenDy data taken on a VERITAs telescope using standard barycentering codes and a radio ephemeris from Jodrell Bank Observatory (Lyne et al. 1993). 

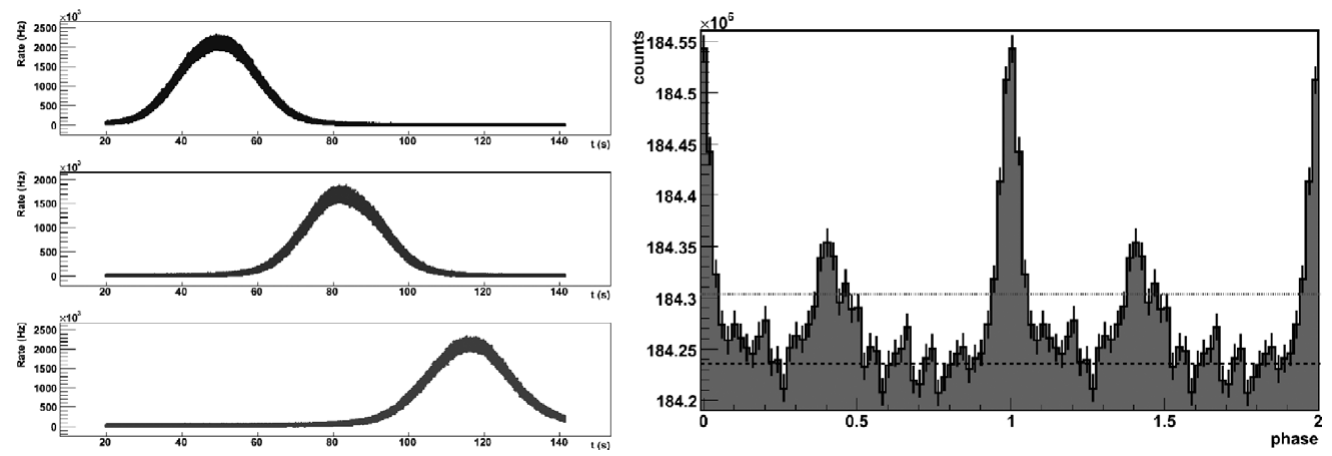

Figure 2. Left: A star is allowed to pass through the field of view of the camera. Three of the seven TRenDy channels are shown here. The count rates from three neighbouring pixels rise and fall as the star passes through their field of view. The scatter is statistical. Right: Phase profile of the Crab Optical Pulsar; two periods are shown to aid the eye. The bottom line is the mean number of counts; the top line represents $5 \sigma$. The sensitivity of TRenDy is $31 \sigma / \sqrt{\text { hour }}$. This data set represents about 45 minutes of data.

\section{Conclusions}

The design for the TRenDy, a multi-channel high-time-resolution photometer, has been outlined, as has its use on VERITAS. Following the success of tests done over the past few months, searches for microsecond optical flares will now take place. Currently under investigation is the possibility of measuring the PMT photocurrents rather than counting pulses as a method of working around the constraints imposed by observing in moonlight.

\section{Acknowledgements}

Special thanks go to my supervisor, David Hanna, for proposing this project, and to Adam Gilbert for his invaluable help in much of the hardware design. TRenDy is supported by grants from NSERC and FQRNT. We acknowledge the excellent work of the technical support staff at the FLWO and at the collaborating institutions in the construction and operation of the instrument.

\section{References}

Bartolini, C., et al. 1994, ApJS 92, 455

Bersier, D., et al. 2003, ApJ 584, L43

Deil, C., et al. 2009, Astropart. Phys. 31, 156

Dolan, J. F. 2001, PASP 113, 974

Fabian, A. C., et al. 1982, A\& A 111, L9

Holder, J., et al. 2006, Astropart. Phys. 25, 391

Kanbach, G., et al. 2001, Nature, 414, 180

Kildea, J., et al. 2007, Astropart. Phys. 28, 182

Lyne, A. G., et al. 1993, MNRAS 265, 1003

Shearer, A., et al. 2003, Science 301(5632), 493

Uemura, M., et al. 2002, PASJ 54, L79 